\title{
Editorial
}

\section{Research in primary care: how to live-up to its needs?}

Primary care has often been represented as the 'coal face of health care' - the place of hard work, slaving under the inescapable demands and realities of daily life. This emphasizes a reality of the care for patients and communities, in that conditions external to health care and medical science determine what can actually be achieved. For many a dedicated primary care provider 'the coal face' metaphorically emphasizes a professional value of engagement in the societal context of patient care, a true 'Name of Honour'. The coal face metaphor is often used in a benign patronizing way by secondary care specialists to express admiration. Implicitly this confirms a picture of primary care at the end of the command chain, receiving orders and instructions from 'above', from the cradle of knowledge with the fuller picture of illness, disease, treatment and care.

The (self)perception of primary care as a mere applicant of medical and health care science has long marred its development and continues to do so today. Primary care takes care of the professional treatment of where the large majority of health problems in the population are located (Green et al., 2001). But although it is widely understood that primary care is essential for effective healthcare (Starfield et al., 2005), investment in its research and development is limited even in countries with sophisticated primary care. In many countries primary care is at best an academic 'teaching' discipline, that distributes knowledge. And 'research' in primary care is often considered 'implementation research' to change the routines of practitioners.

The detrimental implication of the 'coal face' metaphor is that it reinforces a (self)perception that ignores the importance of empirism and its critical reflection, in developing health care. The largest field of patient encounters (Green et al., 2001) must by definition present substantial empirical data.

(C) 2007 Cambridge University Press
The research mission of primary care should be to generate new, not just to implement existing, knowledge.

The World Organization of Family Doctors (Wonca) has defined in the Kingston report (van Weel and Rosser, 2004), the domains of new knowledge for general practice to explore:

1) knowledge of the health problems and their diagnosis and treatment encountered in primary care;

2) navigation of patients through the health care system and the use of health care resources;

3) addressing needs of patients and communities and strengthening of self-efficacy.

Although primary care is broader than general practice, and the role of other primary care disciplines must be strengthened in their own right, these domains cover in all probability primary care at large.

Essential in the Kingston report are the examples of successful primary care research around the world: general practitioners, often in close collaboration with other primary care professionals, have been able to tap into the richness of their daily care of patients and critically appraise it often without much external support from the research community. This should be the positive basis to build a primary care research culture and expertise.

Generating new knowledge aims to improve the health of people and populations around the world. Critical to this is the context in which the knowledge is to be applied - and therefore to be generated. Primary care is defined as health care for individuals in relation to the community in which they live, without restrictions on access with regards to the nature of the health problems, or age, sex or other characteristic of the patient (Wonca Europe, 2002). 'Continuity of care', 'community orientation', 
'patient centeredness' and 'comprehensive care' are key values that shape an all inclusive, horizontal system. But against this approach can be set the principle of restricted primary care, that provides a community directed programme for a single health problem, or for a limited number, for example HIV/AIDS, TB or malaria. Such 'vertical' programming of primary care is particularly prevalent in developing countries, but developed countries are not exempt from it - for example in comprehensive programmes for asthma or COPD.

Where vertical programming involves primary care in the instrumental rolling-out of care, emphasizing the effectiveness of the interventions provided, integrated horizontal primary care adds to it the engagement with communities and the lasting professional relation with individual patients. What science is available points to greater effectiveness and cost-effectiveness of integrated horizontal primary care (Starfield et al., 2005).

Again, these are positive conditions for the development of primary care research: it should base its research on its strength in caring for patients: engagement and lasting professional relations with patients, their families and communities apparently do matter. However, understanding of this process is thus far a black box and it is essential to identify where its strengths and weaknesses lie. And this is where the future of primary care goes: care for aging populations, dominated by chronic diseases, often for patients with co-morbidity or multiple morbidity (van Weel and Schellevis, 2006). This asks for comprehensive multifaceted interventions and a multidisciplinary approach. Research, new knowledge to drive state-of-the-art care can only be generated in primary care. But this requires expertise and a research culture across the board of all disciplines in primary care. The most urgent need is to develop this, to be able to address ambitious multidisciplinary research. This cannot be left to one or a few primary care disciplines, but requires extensive collaboration - of which there are a number of leading examples (Sullivan et al., 2002; The Netherlands School of Primary Care Research, 2006).
This should be the basis to develop evidencebased care for people that responds to needs and makes health care relevant for individuals and communities. This way research will be subservient of the need of equity in health care and provide the academic embedding of general practice and primary care in every community (van Weel, 2006).

\section{Chris van Weel, Professor of General Practice, President-elect World Organization of Family Doctors, Nijmegen, The Netherlands}

\section{References}

Green, L.A., Fryer, G.E., Yawn, B.P., Lanier, D. and Dovey, S.M. 2001: The ecology of medical care revisited. New England Journal of Medicine 344, 2021-25.

Starfield, B., Shi, L. and Macinko, J. 2005: Contribution of primary care to health systems and health. The Milbank Quarterly 83, 457-502.

Sullivan, F.M., Lewison, G. and Clarkson, J. 2002: What Scottish primary care researchers are doing to recover their standing in the UK. Health Bull (Edinburgh) 60,1-4.

The Netherlands School of Primary Care Research (CaRe). Retrieved 7 December 2006, from http.//www. researchschoolcare.nl

van Weel, C. 2006: Towards a GP in every community in the world - promoting health for all through excellence of general practice. The New Generalist.

van Weel, C. and Rosser, W.W. 2004: Improving health care globally: a critical review of the necessity of family medicine research and recommendation to build research capacity. Ann Family Medicine 2, S5-16.

van Weel, C. and Schellevis, F.G. 2006: Comorbidity and guidelines: conflicting interests. Lancet 367, 550-51.

Wonca Europe. 2002: The European definition of general practice/family medicine. Retrieved 7 December 2006. 